\title{
Multi-scale Model-based Vessel Enhancement Using Local Line Integrals
}

\author{
Yuan Yuan and Albert C. S. Chung
}

\begin{abstract}
This paper presents a novel vessel enhancement method. By regarding a vessel segment as a local line and exploiting the second order information along the line, our method embeds a vessel model to capture vessel structures. The vessel model is the key to better performances of our method than the Hessian-based methods and makes the Hessian-based methods fall in an extreme case of our method. It is experimentally shown that our method gives more accurate "vesselness" measures and vessel direction estimations. In particular, our method achieves better background suppression, smoother "vesselness" measures inside vessels and better responses at crossings (where two relatively straight vessels meet).
\end{abstract}

\section{INTRODUCTION}

Vessel enhancement, either as a preprocessing step for vessel segmentation or as a technique to improve visualization of volumetric data, is widely used in computer-aided diagnosis.

The Hessian-based vessel enhancement methods refer to those relying on eigenvalues and/or eigenvectors of the Hessian matrix to distinguish vessels from background; and they are widely used in literature because of the use of second order information to capture local intensity geometry and their high computational efficiency. To the best of our knowledge, Koller et al. [1] were the first to propose to use the Hessian matrix for estimation of vessel directions and to use a matching filter in the direction of vessels to produce a "vesselness" measure. Krissian et al. extended Koller's work in [2]. While the above two works only use eigenvectors of the Hessian matrix, other works [3], [4], [5], [6] use principal curvatures based on eigenvalues of the Hessian matrix. These vessel enhancement filters rely on combinations of eigenvalues of the Hessian matrix to distinguish between vessel structures from blob-like and plane-like structures. Among those works, Frangi's vessel enhancement filtering [4] is extensively used in practice. It uses all three eigenvalues of the Hessian matrix instead of just two eigenvalues [6], [3] and has intuitive geometric interpretations.

However, it is known that second order derivatives are sensitive to local intensity variations. This property is undesirable for vessel enhancement because noise will give false positive responses while crossings (where two relatively straight vessels meet) will produce false negative responses. It is also noticed that responses inside a vessel in noisy regions will tend to be discontinuous. These effects deteriorate visualization results and can create serious problems for the subsequent segmentation methods, especially for

Both authors are with Lo Kwee-Seong Medical Image Analysis Laboratory, Department of Computer Science and Engineering, The Hong Kong University of Science and Technology, Hong Kong \{yyuan, achung\}@ese.ust.hk the commonly used "flux-maximizing geometric flows" by Vasilevskiy et al. [7], which depends greatly on local gradient and hence is sensitive to local intensity variations.

The proposed method in our work embeds a vessel model as prior knowledge by modeling a vessel segment as a local line and exploiting the second order information of the local line integrals in order to capture potentially existing line structures with less sensitivity to local intensity variations and local structure variations. Measures similar to eigenvalues and eigenvectors of the Hessian matrix are produced in a multi-scale fashion and then combined to generate a "vesselness" measure. By the use of this vessel model, our approach is able to give better estimation of vessel directions and better "vesselness" measures. Also, it should be pointed out that the Hessian-based enhancement methods fall in an extreme case of our method.

\section{Methodology}

Given a smooth $\mathcal{N}$-dimensional image $I: \Omega \rightarrow \mathbb{R}, \Omega \subseteq$ $\mathbb{R}^{\mathcal{N}}$, a local line integral transform can be defined as

$$
R^{\vec{v}}[I](x)=\frac{1}{d} \int_{-\frac{d}{2}}^{\frac{d}{2}} I\left(L_{\vec{v}}(t)\right) d t,
$$

where $L_{\vec{v}}(t)=x+t \cdot \vec{v}$ is a line parameterized by $t$, unit vector $\vec{v}$ represents the line direction, $d$ is the integral length and $1 / d$ is a normalizing term. While $R^{\vec{v}}[I]$ gives the intensity average of $I$ along a local line, partial derivative of $R^{\vec{v}}[I]$ gives the average of partial derivatives of $I$.

In particular, for the second derivative,

$$
\frac{\partial^{2}}{\partial x_{i}^{2}} R^{\vec{v}}[I](x)=\frac{1}{d} \int_{-\frac{d}{2}}^{\frac{d}{2}} \frac{\partial^{2}}{\partial x_{i}^{2}} I\left(L_{\vec{v}}(t)\right) d t,
$$

where $x_{i}, i \in\{1,2, \ldots, \mathcal{N}\}$, is a variable for both $I$ and $R^{\vec{v}}$.

Let $H(x)$ be the Hessian matrix for $I$ at point $x$. Following Eqns. 1 and 2, the Hessian matrix $\bar{H}^{\vec{v}}(x)$ for $R^{\vec{v}}[I]$ at point $x$ is then

$$
\bar{H}^{\vec{v}}(x)=\frac{1}{d} \int_{-\frac{d}{2}}^{\frac{d}{2}} H\left(L_{\vec{v}}(t)\right) d t .
$$

Eigenvectors $\bar{e}_{i}$ and corresponding eigenvalues $\bar{\lambda}_{i}$, where $\left|\bar{\lambda}_{1}\right| \leq\left|\bar{\lambda}_{2}\right| \leq \cdots \leq\left|\bar{\lambda}_{\mathcal{N}}\right|, i \in\{1,2, \ldots, \mathcal{N}\}$ of $\bar{H}^{\vec{v}}$ give interesting measures of structures along the local line. Notice that $\bar{e}_{i} \mathrm{~s}$ and $\bar{\lambda}_{i}$ s are functions of $\vec{v}$.

Consider $\mathcal{N}=3$. Let $\lambda_{1}, \lambda_{2}, \lambda_{3}\left(\left|\lambda_{1}\right| \leq\left|\lambda_{2}\right| \leq\left|\lambda_{3}\right|\right)$ be the eigenvalues of $H(x)$ and $e_{1}, e_{2}, e_{3}$ be the corresponding eigenvectors, respectively.

In the Hessian-based methods like [4] and [5], assuming the vessel intensity is brighter than the background intensity, $\left|\lambda_{1}\right| \ll\left|\lambda_{2}\right|$ and $\left|\lambda_{2}\right| \approx\left|\lambda_{3}\right|$ along with $\lambda_{1} \approx 0, \lambda_{2}<0$, $\lambda_{3}<0$, these conditions signal a likely presence of vessel structure at point $x$. Furthermore, $e_{1}$ gives an estimation of the vessel direction. 
At a vessel point $x$, when the line of integration $L_{\vec{v}}(t)$ is aligned with the vessel direction, it will also have the following relationships $\left|\bar{\lambda}_{1}\right| \ll\left|\bar{\lambda}_{2}\right|,\left|\bar{\lambda}_{2}\right| \approx\left|\bar{\lambda}_{3}\right|, \bar{\lambda}_{1} \approx 0$, $\bar{\lambda}_{2}<0, \bar{\lambda}_{3}<0$, which are the same to the relationships as listed above. This is because $\bar{H}^{\vec{v}}$ becomes the average of similar Hessian matrices along $L_{\vec{v}}(t)$ according to Eqn. 3 , given the assumption that intensity structures are similar along the vessel. Furthermore, $\bar{e}_{1}$ will have the same direction as $\vec{v}\left(\bar{e}_{1}= \pm \vec{v}\right)$, the direction of the integral line. Therefore, when $L_{\vec{v}}(t)$ and the vessel are aligned at a vessel point $x,\left|\bar{\lambda}_{2} \bar{\lambda}_{3} \cdot\left\langle\vec{v}, \bar{e}_{1}\right\rangle\right|$ will be maximized. We can obtain an estimation of vessel direction when $\left|\bar{\lambda}_{2} \bar{\lambda}_{3} \cdot\left\langle\vec{v}, \bar{e}_{1}\right\rangle\right|$ is maximized. We denote

$$
\vec{v}^{*}=\arg \max _{\vec{v}}\left|\bar{\lambda}_{2} \bar{\lambda}_{3} \cdot\left\langle\vec{v}, \bar{e}_{1}\right\rangle\right|,
$$

and let $\bar{\lambda}_{i}^{*} \mathrm{~s}$ and $\bar{e}_{i}^{*} \mathrm{~s}$ be the eigenvalues and eigenvectors of $\bar{H}^{\vec{v}^{*}}$, respectively. We can then use $\bar{e}_{i}^{*}$ s to estimate the vessel frame and $\bar{\lambda}_{i}^{*}$ s can be used to distinguish vessels from other undesirable structures similarly as in [4] and [5]. Also, notice that in order to get good estimations, $\vec{v}$ has to uniformly cover the half unit sphere.

\section{A. Vesselness Measure}

Frangi et al. [4] proposed a multi-scale "vesselness" measure to assign a value from 0 to 1 to each point of an image. This assigned value reflects the confidence of a point being inside a vessel. In 3D images, for a single scale $\sigma_{s}$, a response at a point $x$ is calculated as

$$
F_{\sigma_{s}}^{3 D}(x)=\left\{\begin{array}{l}
0 \quad \text { if } \lambda_{2} \geq 0 \text { or } \lambda_{3} \geq 0, \\
\left(1-e^{-\frac{R_{a}^{2}}{2 \alpha^{2}}}\right) e^{-\frac{R_{b}^{2}}{2 \beta^{2}}}\left(1-e^{-\frac{S^{2}}{2 c^{2}}}\right) \text { otherwise, }
\end{array}\right.
$$

where $\lambda_{i} \mathrm{~s}$ are eigenvalues of the Hessian matrix $H(x)$, $R_{a}=\left|\lambda_{2}\right| /\left|\lambda_{3}\right|, R_{b}=\left|\lambda_{1}\right| /\left(\left|\lambda_{2} \lambda_{3}\right|\right)^{\frac{1}{2}}, S=\left(\lambda_{1}^{2}+\lambda_{2}^{2}+\lambda_{3}^{2}\right)^{\frac{1}{2}}$, and $\alpha, \beta, c$ are constant normalization factors. Given a set of scales $\Omega_{\sigma_{s}}$, responses of different scales are combined as $F(x)=\max _{\sigma_{s}}\left\{F_{\sigma_{s}}(x) \mid \sigma_{s} \in \Omega_{\sigma_{s}}\right\}$. A similar "vesselness" measure for $2 \mathrm{D}$ images can be developed as

$$
F_{\sigma_{s}}^{2 D}(x)= \begin{cases}0 & \text { if } \lambda_{2} \geq 0, \\ e^{-\frac{R_{b}^{2}}{2 \beta^{2}}}\left(1-e^{-\frac{S^{2}}{2 c^{2}}}\right) & \text { otherwise, }\end{cases}
$$

where $R_{b}=\left|\lambda_{1}\right| /\left|\lambda_{2}\right|$ and $S=\left(\lambda_{1}^{2}+\lambda_{2}^{2}\right)^{\frac{1}{2}}$.

We use Frangi's "vesselness" measure in this work due to its good performance but we then substitute $\lambda_{i} \mathrm{~s}$ with $\bar{\lambda}_{i}^{*} \mathrm{~s}$ to reduce the sensitivity to local intensity variations. In all the experiments presented in this paper, parameters $\alpha, \beta$ were set to 0.5. $c$ was set to half of $\max \{S(x) \mid x \in \Omega\}$ for both Frangi's and our method.

It should also be noted that, same as Frangi's method, we use the concept of normalized derivatives proposed by Lindeberg [8] to deal with multi-scale normalization. Therefore, numerical differentiation of an image at a scale $\sigma_{s}$ is defined as $\frac{\partial}{\partial x_{i}} I_{\sigma_{s}}=\sigma_{s}^{\gamma} \cdot I * \frac{\partial}{\partial x_{i}} G_{\sigma_{s}}$, where $\gamma$ is a normalizing term. In this work, $\gamma$ was set to 1 for all methods.

\section{B. Vessel Model as Prior Knowledge}

By probing all local lines of length $d$, our method can determine whether a point belongs to a vessel based on more information than mere local intensity structures. At crossings, since local line structures are also present, the proposed method improves responses when the selected integral line is long enough to cover the crossing and extend to vessel portions that are not on the crossing. This reduces false negatives that the Hessian-based enhancement methods produce.

The choice of $d$ depends on image resolution and curvatures of vessels in the image. If $d$ is too large, responses at vessel structures with high curvature will decline. On the other hand, if $d$ is too small, noise will not be effectively reduced and response at junctions will still be low.

\section{Hessian as Extreme Case}

It is interesting to notice that, for any unit vector $\vec{v}$, $\lim _{d \rightarrow 0} \bar{H}^{\vec{v}}(x)=H(x)$, Therefore, taking limit $d \rightarrow 0$, $\bar{\lambda}_{i}^{*}=\lambda_{i}$ and $\bar{e}_{i}^{*}=e_{i}$ for $i=\{1,2, \ldots, \mathcal{N}\}$. Hence, the conventional Hessian-based enhancement methods fall in an extreme case of the proposed method.

\section{EXPERIMENTAL RESUlts}

In this section, we compare our method with two other vesselness filters proposed by Frangi et al. [4] and $\mathrm{Li}$ et al. [5]. The evaluations include enhancement results on all 2D retinal images from the DRIVE database [9] and a 3D time-of-flight magnetic resonance angiographic (TOF-MRA) image. Both qualitative and quantitative results are shown.

The reason why Frangi's method was chosen to compare with is its extensive use in practice and its better performance than most of the other methods due to its efficient use of eigenvalues of the Hessian matrix. Li's method was also chosen as a representative of other vessel enhancement filters based on eigenvalues.

The reported filters above use eigenvalues $\left|\lambda_{1}\right| \leq\left|\lambda_{2}\right| \leq$ $\left|\lambda_{3}\right|$ of the Hessian matrix. Our method differs from Frangi's method in the use of $\bar{\lambda}_{i}^{*}$ s. Frangi's method has an different "vesselness" measure from Li's method.

In [5], Li et al. proposed a different measure. The response $L_{\sigma_{s}}^{3 D}(x)$ for a single scale $\sigma_{s}$ is given by

$$
L_{\sigma_{s}}^{3 D}(x)=\left\{\begin{array}{ll}
0 & \text { if } \lambda_{2} \geq 0 \text { or } \lambda_{3} \geq 0, \\
\frac{\left|\lambda_{2}\right|\left(\left|\lambda_{2}\right|-\left|\lambda_{1}\right|\right)}{\left|\lambda_{3}\right|} & \text { otherwise }
\end{array} .\right.
$$

$$
\begin{aligned}
& \text { A 2D version can be formed as } \\
& \qquad L_{\sigma_{s}}^{2 D}(x)=\left\{\begin{array}{ll}
0 & \text { if } \lambda_{2} \geq 0, \\
\left|\lambda_{2}\right|-\left|\lambda_{1}\right| & \text { otherwise }
\end{array} .\right.
\end{aligned}
$$

\section{A. Benchmarks}

Let $\Omega$ be the image domain and $\Omega_{v} \subseteq \Omega$ be the vessel region, given the ground truth of an image $I^{*}(x)=$ $\left\{\begin{array}{l}1 \quad \text { if } x \in \Omega_{v}, \\ 0 \text { otherwise, }\end{array}\right.$ and $V^{*}: \Omega_{v} \rightarrow \mathbb{R}^{2}$, the true vessel directions, an angular discrepancy measure can be defined as

$$
A D=\frac{1}{N_{v}} \sum_{x \in \Omega_{v}}\left|\left\langle V(x),\left[\begin{array}{cc}
0 & -1 \\
1 & 0
\end{array}\right] V^{*}(x)\right\rangle\right|,
$$

where $N_{v}=\left|\Omega_{v}\right|, V(x)$ is the estimated vessel direction at point $x$. The more consistent between the estimated directions and the true directions are, the less angular discrepancy there will be. Also, $L_{1}$ norm is used to measure the errors from the rescaled enhanced results to $I^{*}$.

$$
\left\|\tilde{E}-I^{*}\right\|_{L_{1}}=\frac{1}{N} \sum_{x \in \Omega}\left|\left(\tilde{E}(x)-I^{*}(x)\right)\right|,
$$


where $N=|\Omega|$, and $\tilde{E}$ is the rescaled result to $[0,1]$. We use these two benchmarks to evaluate performances of different enhancement methods.

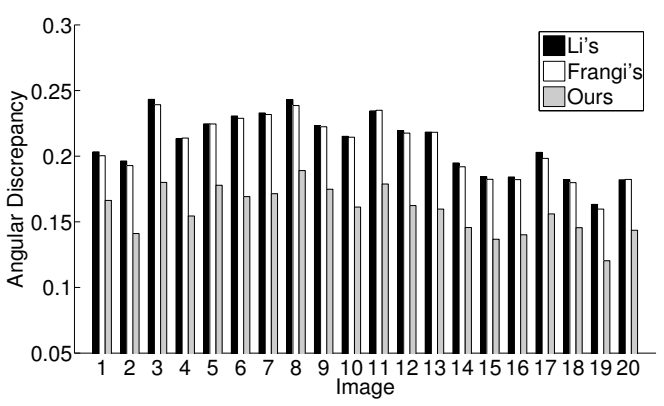

(a)

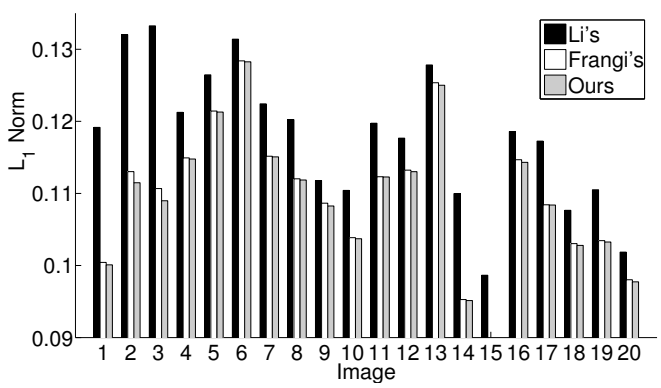

(b)

Fig. 1. (a) Angular discrepancy of each method for different images. (b) $L_{1}$ norm of errors of each method for different images.

\section{B. 2D Retinal Images}

We tested our method against the other two methods on a publicly available retinal image database, DRIVE [9]. All images in the DRIVE database were tested. In the experiments, our method gives promising results that the enhancement performance can be improved, especially for low contrast and noisy vessels. We set $\Omega_{\sigma_{s}}=\{0.5,1,2,2.4,3\}$ for smoothing the input images for all methods, and $d$ was set to 7 for our method. $\vec{v}$ was set to have an angular resolution of $10^{\circ}$. Fig. 1 shows the angular discrepancy and $L_{1}$ norm of errors for all 20 testing images in the DRIVE database, which provides manually segmented results to serve as the ground truths.

Fig. 1 shows that our method provides the lowest angular discrepancy and $L_{1}$ norm of errors among all three methods. While Fig. 1a shows that our method significantly improves vessel direction estimations than the other two methods, it is noticed in Fig. 1b, $L_{1}$ norm of errors of our results are comparable to those of Frangi's method. It is because vessels in noisy and low contrast regions where our method surpasses the other two methods give quite low responses when rescaled to $[0,1]$, since there are particularly strong vessels in each image in the DRIVE database. Besides, crossings only occupy a small portion of the entire image.

Figs. 2 and 3 show two regions of interest (ROI) along with enhancement responses for each method and the ground truths. Estimated vessel directions scaled by "vesselness" are also shown. It is observed that our method produces accurate vessel direction estimations and responses. In particular, our method has smoother measures inside vessels and is more robust against noise. Also, it is shown in Fig. 3 that our method has a better response at the crossing.

\section{C. $3 D$ Real Images}

One 3D time-of-flight magnetic resonance angiographic (TOF-MRA) brain image volume, obtained from the University Hospital of Zurich, Switzerland, with the size of $512 \times 512 \times 100$ voxels and voxel size $0.35 \mathrm{~mm} \times 0.35 \mathrm{~mm} \times$ $0.75 \mathrm{~mm}$, was enhanced using all three methods. The comparisons are shown as maximum intensity projections (MIP). $d=7$ for our method. The set of sample directions $\vec{v} \mathrm{~s}$ are taken as $\{\vec{v}=[\cos \phi \cos \theta, \cos \phi \sin \theta, \sin \phi] \mid \theta, \phi \in$ $\left.\left\{0^{\circ}, 10^{\circ}, \ldots, 170^{\circ}\right\}\right\}$.

Results from our method are shown in Figures 4(d). Comparing with those results from the Li's method (Figure 4(b)) and the Frangi's method (Figure 4(c)), better background suppression obtained from our method is observed while small vessels are also preserved.

\section{CONCLUSION}

In this paper we have presented a novel vessel enhancement technique. The main contributions of this work can be summarized as follows:

- A vessel model is embedded as prior knowledge by regarding a vessel segment as a local line and using the second order information of the local line integrals. The proposed method is less sensitive to local intensity variations and local structure variations. Therefore, it can produce more accurate "vesselness" measures and vessel direction estimations.

- Better vessel directions and "vesselness" measures generated by our method can benefit various applications, like vessel tracking and vessel segmentation.

- The vessel model in the proposed method is general in nature. It is shown that the Hessian-based enhancement methods fall in an extreme case of the proposed method.

\section{REFERENCES}

[1] T. Koller, G. Gerig, G. Szekely, and D. Dettwiler, "Multiscale detection of curvilinear structures in 2-D and 3-D image data," ICCV, pp. 864$869,1995$.

[2] K. Krissian, G. Malandain, N. Ayache, R. Vaillant, and Y. Trousset, "Model based multiscale detection of 3D vessels," CVPR, pp. 722727, 1998.

[3] Y. Sato, S. Nakajima, N. Shiraga, H. Atsumi, S. Yoshida, T. Koller, G. Gerig, and R. Kikinis, "Three-dimensional multi-scale line filter for segmentation and visualization of curvilinear structures in medical images," MedIA, vol. 2, no. 2, pp. 143-168, 1998.

[4] A. Frangi, W. Niessen, K. Vincken, and M. Viergever, "Multiscale vessel enhancement filtering," MICCAI, pp. 130-137, 1998.

[5] Q. Li, S. Sone, and K. Doi, "Selective enhancement filters for nodules, vessels, and airway walls in two-and three-dimensional CT scans," Medical Physics, vol. 30, pp. 20-40, 2003.

[6] C. Lorenz, I.C. Carlsen, T.M. Buzug, C. Fassnacht, and J. Weese, "Multi-scale line segmentation with automatic estimation of width, contrast and tangential direction in 2D and 3D medical images," CVRMed-MRCAS, pp. 233-242, 1997.

[7] A. Vasilevskiy and K. Siddiqi, "Flux maximizing geometric flows," PAMI, vol. 24, no. 12, pp. 1565-1578, 2002.

[8] T. Lindeberg, "Edge detection and ridge detection with automatic scale selection," IJCV, vol. 30, no. 2, pp. 117-154, 1998.

[9] J. Staal, MD Abramoff, M. Niemeijer, MA Viergever, and B. van Ginneken, "Ridge-based vessel segmentation in color images of the retina," TMI, vol. 23, no. 4, pp. 501-509, 2004. 


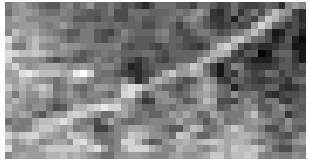

(a)

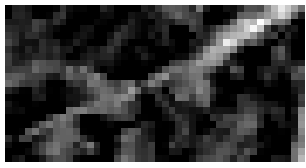

(b)

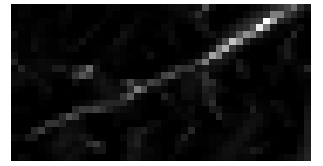

(c)

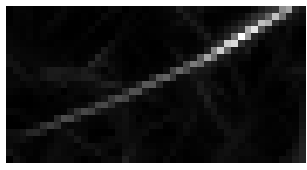

(d)

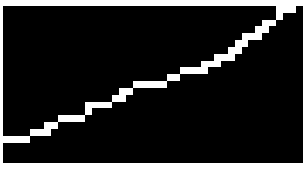

(e)

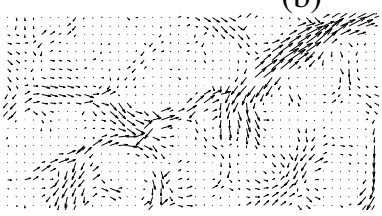

(f)

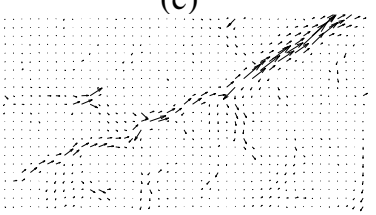

(g)

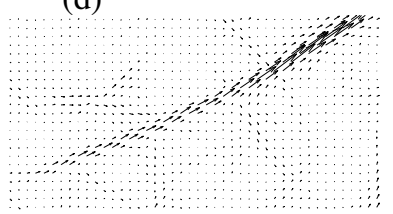

(h)

Fig. 2. (a)ROI. Enhancement results obtained by using (b) Li's method, (c) Frangi's method, and (d) the proposed method with $d=7$. (e) Ground truth of ROI. (f),(g),(h) Estimated vessel directions scaled by corresponding "vesselness" measures from Li's, Frangi's and the proposed method respectively.

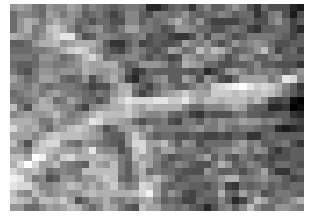

(a)

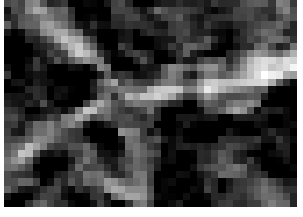

(b)

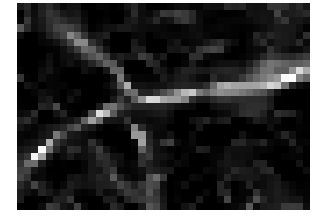

(c)

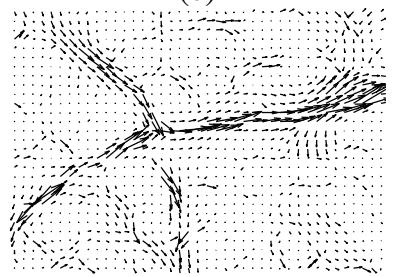

(g)

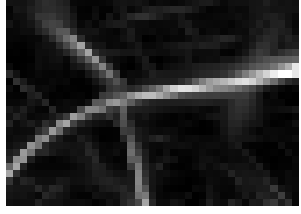

(d)

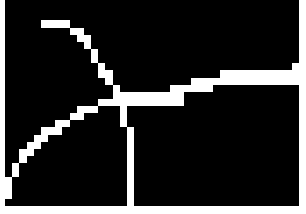

(e)

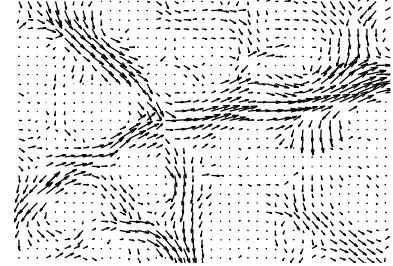

(f)

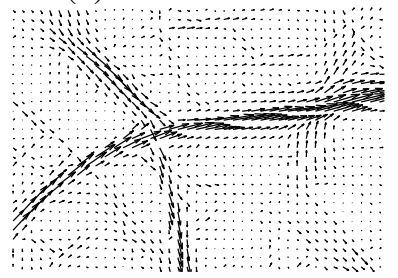

(h)

Fig. 3. (a)ROI. Enhancement results obtained by using (b) Li's method, (c) Frangi's method, and (d) the proposed method with $d=7$. (e) Ground truth of ROI. (f),(g),(h) Estimated vessel directions scaled by corresponding "vesselness" measures from Li's, Frangi's and the proposed method respectively.

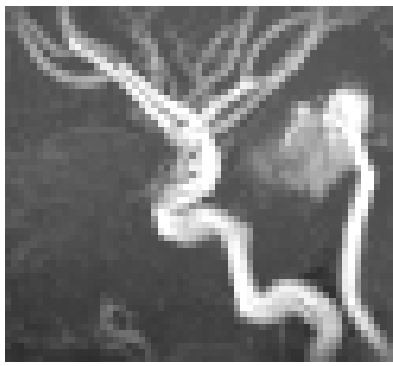

(a)

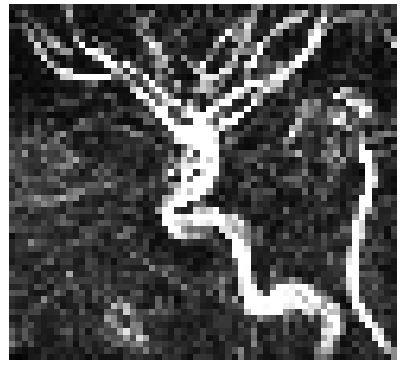

(b)

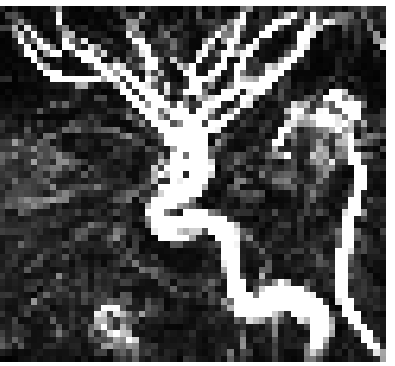

(c)

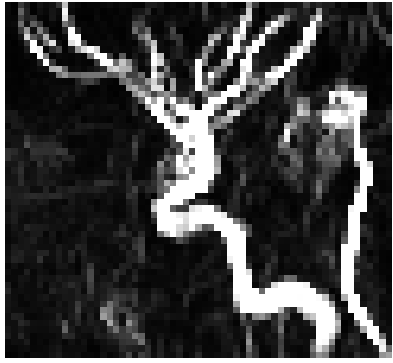

(d)

Fig. 4. (a) Sagittal MIP of ROI. Enhancement results obtained by using (b) Li's method, (c) Frangi's method, and (d) the proposed method with $d=7$. (b) (c) (d) are rescaled to $[0, .5]$ for better illustration of noise level. 